\title{
Olbers' paradox has more to teach
}

The question of why the sky is dark at night is a textbook problem, but textbook answers may still miss the point. The problem is that of determining which is the more dominant of the two preferred solutions.

Over the years, Olbers' paradox has become a kind of classic - it is a real paradox, which everybody knows can be resolved in an interesting way. Most often, the paradox and its resolution are advanced as evidence that the theory of relativity is not merely compatible with the night sky that we know, but that it is essential in accounting for its appearence. But even paradoxes, it appears, are not always what they seem.

The issue is simply that of why the night sky is not uniformly as bright as the surface of the Sun. That, after all, is what one would expect if the stars in the Universe number something like infinity, or at least if there are so many of them that their collective surface area projected on the sphere of the sky would suffice to cover it completely - or to tile it, in the technical sense.

Then the output of visible radiation from any element of the night sky would be more or less identical with that from an equal area of the surface of the Sun. Of course, there would be occasions when cool stars would be interposed between ourselves and brighter but more distant stars, but that would merely give the night sky a granular appearance. In general, we would have the uncomfortable impression that we live at the centre of a hollow black body whose temperature is about 6,000 degrees centigrade.

The question why the real world is not like that is Olbers' paradox. And a little reflection will show that some obvious resolutions will not suffice. If, for example, the Universe is supposed to be uniformly populated by stars like the Sun, filling the Universe uniformly with obscuring dust will not get round the difficulty. The intensity of the radiation from a distant star will decrease exponentially with its distance, but cannot do so quickly enough to resolve the paradox without also obscuring the Sun itself. And what, in any case, would be the fate of such a dust-filling in a black-body oven of this kind?

Arguments like that explain why the resolution of the paradox must be in some way cosmological. In simple language, there are two ways of doing the trick. First, if the galaxies that house the distant stars are finite in age, there has only been a finite time during which they can have been producing radiation; the radiation field in the black-body oven may be destined to reach the typical surface temperature of a star, but there has not yet been time enough for the oven to reach equilibrium.

Second, and more appealing, if the Universe is expanding, the radiation from distant stars is shifted towards the red end of the spectrum, while the volume of the Universe that must be filled with radiation is continually and continuously increasing. These, of course, are the arguments that make Olbers' paradox a part of the case for believing in relativity.

The two arguments are, of course, distinct, even though they may be linked together in some views of what the Universe is like. So which of them should carry the greater weight? Paul S. Wesson from the University of California at Berkeley and from the University of Waterloo at Toronto has for some years been conducting a personal crusade to rid the world of erroneous resolutions of the paradox. His latest essay in this direction, in the 1 February issue of the Astrophysical Journal $(367,399 ; 1991)$ reiterates the conclusion of his earlier arguments that it is the finite age of the galaxies, and not the expansion of the Universe, that chiefly accounts for the resolution of the paradox.

The question of which effect is the more important is necessarily quantitative. Both effects must have some influence; the issue is that of which influence is the greater. The result is that the finite age of the galaxies accounts for a reduction by a factor of ten in the intensity of intergalactic radiation (which Wesson calls extragalactic background light), and that the relativistic effects, notably the expansion of the Universe, account for a factor of only three or thereabouts.

Amusingly, Wesson starts with an account of how the textbooks handle (or mishandle) Olbers. Most of the textbooks, he says, are "still less than satisfactory" even though the "situation is better now". Wesson's article, in the circumstances, may properly be regarded as a kind of book review on one technical issue, but the paper also has a calculation of the extragalactic radiation field to be expected with different models of the expanding Universe. He writes with something of the resignation of one saying the last word on a topic for the umpteenth time.

But how does one set about calculating the expected radiation field? It is not, of course, a trivial problem. Allowing for the shifting of the radiation towards the red end of the spectrum is easily accomplished for a particular model of the Universe, but it is also necessary to allow for the mode of evolution of the galaxies, which are generally supposed to have evolved from hotter to cooler, but in some way that cannot easily be determined. (If it were otherwise, the big problems of cosmology would surely have been solved a long time ago.)

For Wesson's purpose, that of calculating an order of magnitude, it is sufficient to suppose that the average temperature of a galaxy is a decreasing function of time, and to use that as a basis for calculating the output of radiation from a galaxy using the familiar Planck formula for the spectral intensity of the radiation from a black body.

With the help of some remarkably straightforward algebra, Wesson derives an expression for the light intensity from an ensemble of galaxies which depends on the two effects with which he is principally concerned in distinct ways. The finite age of the galaxies enters by means of the lower limit of an integral over time, while the strictly relativistic effects arise through a scaling factor determined by the relativistic model of the expansion.

The virtue of this piece of algebra is that it makes it possible to compare the sensitivity of the radiation to the two effects the finite age of the galaxies and the relativistic expansion - without having to be numerically sure about all of the other details.

In the end, the comparison is made even less dependent on the values of irrelevant parameters by the device of directly comparing the intensity of the intergalactic radiation field in an expanding and a static model of the Universe. What Wesson finds is that the ratio rarely exceeds $1: 3$, and is often much less. By contrast, there are numerical arguments to suggest that the effect of the finite galactic age will be greater.

In the circumstances, it hardly seems appropriate that people should go on telling students that the resolution of Olbers' paradox is a relativistic effect. It is more relevant that there was a time in the history of the Universe when there were no galaxics of the form in which we know them, and thus no opportunity to fill the Universe with radiation of the kind now called visible. What happened before then, of course, remains anybody's guess.

John Maddox 